\title{
Efficient tool to calculate two-dimensional optical spectra for photoactive molecular complexes
}

\author{
Hong-Guang Duan, ${ }^{1,2}$ Arend G. Dijkstra, ${ }^{2}$ Peter Nalbach, ${ }^{1,3}$ and Michael Thorwart ${ }^{1,3, *}$ \\ ${ }^{1}$ I. Institut für Theoretische Physik, Universität Hamburg, Jungiusstraße 9, 20355 Hamburg, Germany \\ ${ }^{2}$ Max Planck Institute for the Structure and Dynamics of Matter, Luruper Chaussee 149, 22761 Hamburg, Germany \\ ${ }^{3}$ The Hamburg Centre for Ultrafast Imaging, Luruper Chaussee 149, 22761 Hamburg, Germany
}

(Received 25 June 2015; published 13 October 2015)

\begin{abstract}
We combine the coherent modified Redfield theory (CMRT) with the equation of motion-phase matching approach (PMA) to calculate two-dimensional photon-echo spectra for photoactive molecular complexes with an intermediate strength of the coupling to their environment. Both techniques are highly efficient, yet they involve approximations at different levels. By explicitly comparing with the numerically exact quasiadiabatic path integral approach, we show for the Fenna-Matthews-Olson complex that the CMRT describes the decay rates in the population dynamics well, but final stationary populations and the oscillation frequencies differ slightly. In addition, we use the combined CMRT+PMA to calculate two-dimensional photon-echo spectra for a simple dimer model. We find excellent agreement with the exact path integral calculations at short waiting times where the dynamics is still coherent. For long waiting times, differences occur due to different final stationary states, specifically for strong system-bath coupling. For weak to intermediate system-bath couplings, which is most important for natural photosynthetic complexes, the combined CMRT+PMA gives reasonable results with acceptable computational efforts.
\end{abstract}

DOI: 10.1103/PhysRevE.92.042708

PACS number(s): 87.80.Dj, 03.65.Yz, 03.65.Ud, 03.65.Ta

\section{INTRODUCTION}

Photosynthesis is the process used by plants and bacteria to convert the energy of sunlight into chemical energy in order to fuel the organism's activities. In the initial steps of the photosynthetic process, pigment-protein complexes complete the light-energy transfer and charge separation with a near unity quantum efficiency. Light-harvesting molecular complexes achieve the energy transfer by using an array of light-harvesting pigments that absorb the energy to form excitons and funnel the excitation to the reaction center [1]. To investigate the energy transfer dynamics in the first steps of photosynthesis on the fs time scale, ultrafast spectroscopic tools are available. Among the techniques used, two-dimensional (2D) photon-echo spectroscopy is a powerful tool, which allows for direct mapping of the excitation energy pathways as a function of absorption and emission wavelength [2]. It is particularly useful in examining photosynthetic systems in which the manifold of electronic states is closely spaced and broadening through static disorder yields highly congested spectra. Recent experimental 2D electronic spectroscopic studies of the Fenna-Matthews-Olson (FMO) complex observed coherent beating signals and, thus, raised interest in the interplay between energy transfer, long-lived quantum coherence in photosynthetic processes [3], and low-frequency vibrations of the molecular back bone. Also in photoactive marine cryptophyte algae [4], the light-harvesting complex LH2 [5] of rhodobacter sphaeroides, and in the reaction center [6,7] of the Photosystem II, long-lived oscillations have been experimentally observed at low $(77 \mathrm{~K})$ and room temperatures $(300 \mathrm{~K})$, indicating a strong vibronic coupling in these systems.

To analyze the experimental findings in such large and complex photoactive molecular complexes, a thorough comparison

*michael.thorwart@physik.uni-hamburg.de with theoretical calculations is essential, in order to arrive at a reliable interpretation of the measured 2D spectra. Since it is a difficult and computationally demanding task to determine 2D optical spectra, often only the population dynamics of exciton states is calculated. For the FMO complex, a rather small light-harvesting complex, the hierarchical equations of motion [8] were applied and quantum oscillations were observed on the time scale of the 2D experiments employing an environmental Debye model spectral density with rather small reorganization energy [9]. Employing the numerically exact quasiadiabatic propagator path integral (QUAPI) allowed us to use a more realistic measured environmental spectral density. However, this resulted in a decay of the decoherence faster than experimentally observed $[10,11]$. This spectral density could, more recently, be employed to calculate the 2D spectra of FMO with the hierarchy equation [12] and a reasonable agreement between theory and experiment could be achieved. The calculations of QUAPI and the hierarchical equations of motion treated the coupling of the complex to environmental fluctuations numerically exactly. However, the computational effort is immense, which makes the simulation of larger lightharvesting molecular complexes (which contain, typically, dozens to hundreds of excitonic subunits) virtually impossible. The need for a highly efficient numerical tool to calculate 2D optical spectra of large molecular complexes with a reasonable numerical effort and a satisfactory accuracy still exists and it is expected to continue to increase.

Given their complex molecular structures, for the calculation of 2D spectra of large light harvesters, approximate schemes are usually unavoidable. Standard Redfield equations [13], which invoke a lowest-order Born and a Markovian approximation, are good at weak system-bath coupling, but fail for strong coupling. The regime of intermediate system-bath coupling as present for the exciton dynamics in photoactive complexes [14] is typically also not properly treated within Redfield equations [15-19]. Thus, the modified Redfield theory (MRT) has been widely used for the description 
of energy transfer processes of large molecules [20-26]. This approach includes that contribution of the system-bath coupling Hamiltonian, which is diagonal in the eigenbasis of the system. In turn, it includes the off-diagonal terms of the system-bath coupling Hamiltonian in the form of a second-order perturbative approximation. The equation of motion of the MRT only includes the population transfer within the reduced density matrix, but the accompanying dephasing is not presented. The accuracy of the MRT in view of the dynamics of the reduced density matrix has been analyzed in detail [27]. Moreover, MRT has been shown to have a somewhat wider range of applicability when compared to both the original Redfield and Förster theory [21]. Also, linear absorption spectra for an ensemble of B850 rings have been determined, which shows that MRT includes non-Markovian effects, which clearly show up in the high-energy part of the static absorption line shapes [28]. Different energy transfer components of LHCII trimer and phycoerythrin 545 have been revealed using MRT by simultaneous quantitative fits of the absorption, linear dichroism, steady-states fluorescence spectra, and transient absorption kinetics upon excitation at different wavelengths [29].

A more refined description of the quantum dissipative exciton dynamics is achieved in this work upon observing that the electronic dephasing, which is induced by the populationtransfer, can be efficiently included in the quantum master equation. The off-diagonal terms in the quantum master equation now include the decoherence of excited states and electronic dephasing between ground and excited states by exploiting the relation $1 / T_{2}=1 / 2 T_{1}+1 / T_{2}^{*}$ to estimate the different contributions to the dephasing rate. Here, $T_{2}$ is the transverse relaxation time and $T_{1}, T_{2}^{*}$ are the longitudinal relaxation time and pure dephasing time, respectively. While working out the details with the results reported in this paper, this extended quantum master equation has also been independently put forward very recently in Ref. [30] and has been named the coherent modified Redfield theory (CMRT). To avoid confusion, we use to this nomenclature also here.

For calculating 2D photon-echo spectra, essentially two different approaches are available. On the one hand, the response to the sequence of applied laser pulses can be calculated by evaluating the third-order optical response function [31]. Modified Redfield theory was successfully applied to simulate the 2D spectra of the double-ring LH2 aggregate of purple bacteria including both the B800 and the B850 ring [32]. Comparing experimentally measured and theoretically calculated results of 2D spectra revealed that excitation energy transfer through the LHCII happens on three time scales: sub-100fs relaxation through spatially overlapping states, several hundred femtosecond transfer between nearby chlorophylls, and picosecond energy transfer steps between layers of pigments [33]. More recently, 2D spectra of the reaction center in the Photosystem II were calculated with MRT at low temperature, and the charge separation process was investigated by MRT including charge transfer states in the model $[22,34]$.

An alternative approach to calculate 2D optical spectra, which is especially useful when finite durations of the laser pulses as well as pulse overlap effects are taken into account, is the equation of motion phase matching approach
(PMA) [35,36]. Using the PMA in combination with the conventional Redfield equations, 2D spectra of a single FMO subunit were studied, and the signature of energy transfer was revealed by well-resolved peaks in the simulation with adjustable pure dephasing of exciton states [37].

Although MRT is used to tackle many different problems in the study of energy transport in photosynthetic complexes, no investigation of its reliability in calculating nonlinear and, specifically, 2D optical spectra is at hand. In this work, we first verify the CMRT approach by comparing the population dynamics of FMO exciton states with numerically exact results of the QUAPI approach. In addition, we combine the CMRT with the PMA to calculate 2D photon-echo spectra for a simple dimer model. Again, the results of CMRT+PMA are benchmarked against numerically exact results of the QUAPI approach. For the long-time steadystate dynamics, the CMRT+PMA and QUAPI simulations show differences for intermediate and strong system-bath coupling. However, for intermediate coupling, as it is typical in photosynthetic complexes, the short-time dynamics including dephasing times and coherent beating frequencies are well described by CMRT+PMA. Hence, an efficient numerical scheme to calculate $2 \mathrm{D}$ photon-echo spectra with a reasonable computational effort is available.

The remainder of this paper is organized in the following way. In Sec. II, we briefly introduce the model of the FMO complex for which we compare the performance of the CMRT+PMA in calculating a nontrivial population dynamics. Additionally, the dimer model is introduced for which we compare results of $2 \mathrm{D}$ photon-echo spectra. A brief description of the CMRT+PMA and QUAPI for calculating the reduced density matrix and 2D spectra is given in Sec. III. In Sec. IV, we give the results of the comparison, and a thorough discussion is appended in Sec. V, before we finish with a conclusion.

\section{MODEL}

In the framework of open quantum systems the Hamiltonian of the complete system $H$ can be decomposed as four parts

$$
\begin{aligned}
H & =H_{S}+H_{S B}+H_{B}+H_{\mathrm{ren}}, \\
H_{S} & =\sum_{m=1}^{N} \epsilon_{m} a_{m}^{\dagger} a_{m}+\sum_{m=1}^{N} \sum_{n<m} J_{n m}\left(a_{m}^{\dagger} a_{n}+a_{n}^{\dagger} a_{m}\right), \\
H_{B} & =\sum_{m=1}^{N} \sum_{j=1}^{N_{b}^{m}}\left(\frac{p_{m j}^{2}}{2}+\frac{1}{2} \omega_{m j} x_{m j}^{2}\right),
\end{aligned}
$$

where $\epsilon_{m}$ is the on-site transition energy and $J_{n m}$ is the intermolecular coupling. $N$ is the total number of monomers. $N_{b}^{m}$ is the number of bath modes coupled to molecule $m$, which we will take to be infinity. $x_{m j}$ and $p_{m j}$ are the mass weighted position and momentum of the $j$ th harmonic oscillator bath mode with frequency $\omega_{m j}$. The interaction term $H_{S B}=\sum_{m} K_{m} \Phi_{m}(x)$ induces the coupling between system and bath. It is assumed to be separable such that $K_{m}$ only acts on the system subspace and $\Phi_{m}(x)$ only on the bath degrees of freedom. In the following we further assume a linear relation between bath coordinates and the system. The system-bath 
interaction is then given as

$$
H_{S B}=\sum_{m} K_{m} \sum_{j} c_{m j} x_{m j}
$$

and we furthermore restrict our considerations to pure electronic dephasing only, i.e. $K_{m}=a_{m}^{\dagger} a_{m}$. The renormalization term is

$$
H_{\mathrm{ren}}=\sum_{m} \sum_{j} K_{m}^{2} \frac{c_{m j}^{2}}{m_{m j} \omega_{m j}^{2}} .
$$

This term compensates for artificial shifts of the system frequencies due to the system-bath interaction.

The influence of the bath is fully described by its bath spectral density

$$
J_{m}(\omega)=\pi \sum_{j} \frac{c_{m j}}{2 m_{m j} \omega_{m j}} \delta\left(\omega-\omega_{m j}\right)=2 \lambda \frac{\omega \gamma}{\omega^{2}+\gamma^{2}}
$$

with reorganization energy $\lambda$ and high-frequency cutoff $\gamma$. We assume the bath at each monomer to be independent (no cross correlation between baths [38]) but with identical spectra, i.e., $J_{n}(\omega)=J_{m}(\omega)$.

Laser pulses acting on the exciton complex result in the addition of a system-field interaction, i.e., $H_{S} \rightarrow H_{S}+F(t)$, which is defined within the dipole approximation according to

$$
F(t)=-X \mathbf{E}(t)+\text { H.c. },
$$

with the electric field of the laser pulse $\mathbf{E}(t)$ and the electronic transition dipole operator $\boldsymbol{\mu}$ of the exciton system

$$
\boldsymbol{\mu}=X+X^{\dagger} \quad \text { with } \quad X=\sum_{m=1}^{N} \boldsymbol{\mu}_{m} a_{m} .
$$

$\boldsymbol{\mu}_{m}$ determines the dipole strength and direction of the $m$ th monomer.

\section{A. Dimer}

The dimer is modeled by two monomers with site energies $\epsilon_{1}=-50 \mathrm{~cm}^{-1}=-\epsilon_{2}$ and a coupling $J=150 \mathrm{~cm}^{-1}$. The two dipole moments are considered to be perpendicular to each other, i.e., $\boldsymbol{\mu}_{1} \perp \boldsymbol{\mu}_{2}$. For the bath spectral density as specified in Eq. (4), we choose $\lambda=50 \mathrm{~cm}^{-1}$ and $100 \mathrm{~cm}^{-1}$, and set $\gamma=100 \mathrm{~cm}^{-1}$.

\section{B. FMO}

We model a monomer subunit of the FMO trimer by including seven bacteriochlorophyll $a$ molecular sites. The single excitation subspace is described by a Hamiltonian

$$
H_{F}=\left(\begin{array}{rrrrrrr}
240 & -87.7 & 5.5 & -5.9 & 6.7 & -13.7 & -9.9 \\
& 315 & 30.8 & 8.2 & 0.7 & 11.8 & 4.3 \\
& & 0 & -53.5 & -2.2 & -9.6 & 6.0 \\
& & & 130 & -70.7 & -17.0 & -63.3 \\
& & & & 285 & 81.1 & -1.3 \\
& & & & & 435 & 39.7 \\
& & & & & & 245
\end{array}\right)
$$

in units of $\mathrm{cm}^{-1}$, where we use the site energies and dipolar couplings determined by Adolphs and Renger [14] for the
FMO complex of Chlorobium tepidum. Bath parameters are chosen as $\lambda=35 \mathrm{~cm}^{-1}$ and $\gamma=53 \mathrm{~cm}^{-1}$ following Ref. [8].

\section{METHODS}

\section{A. Coherent modified Redfield theory (CMRT)}

The CMRT can be derived from the Nakajima-Zwanzig equation [28] using a scheme for the separation of the total Hamiltonian, which does not treat the whole system-bath interaction term $H_{S B}$ perturbatively [15,39]. Instead, the Hamiltonian is separated according to

$$
\begin{aligned}
& H_{0}=H_{S}+H_{B}+\sum_{\mu}|\mu\rangle\left\langle\mu\left|H_{S B}\right| \mu\right\rangle\langle\mu|, \\
& H^{\prime}=\sum_{\mu \nu, \mu \neq v}|\mu\rangle\left\langle\mu\left|H_{S B}\right| \nu\right\rangle\langle\nu|,
\end{aligned}
$$

where $|\mu\rangle$ are eigenstates of $H_{S}$ and $H^{\prime}$ is the off-diagonal term of the system-bath interaction part in the exciton basis. In this basis, $H_{0}$ is diagonal and the matrix elements read

$$
\left\langle\mu\left|H_{0}\right| \mu\right\rangle=\epsilon_{\mu}-\lambda_{\mu \mu \mu \mu}+H_{B}(\mu),
$$

where $\epsilon_{\mu}$ is the $\mu$ th excitonic level of the system Hamiltonian and

$$
\lambda_{\mu \nu \mu^{\prime} v^{\prime}}=\sum_{m}\left\langle\mu\left|K_{m}\right| v\right\rangle\left\langle\mu^{\prime} \mid K_{m}\right\rangle v^{\prime} \sum_{j} \frac{c_{m j}^{2}}{2 m_{m j} \omega_{m j}^{2}}
$$

is the weighted reorganization energy. Moreover,

$$
H_{B}(\mu)=\frac{1}{2} \sum_{\xi}\left[\frac{p_{\xi}^{2}}{m_{\xi}}+m_{\xi} \omega_{\xi}^{2}\left(x_{\xi}+\sum_{k} \frac{\left\langle\mu\left|K_{k}\right| \mu\right\rangle}{m_{\xi} \omega_{\xi}^{2}}\right)^{2}\right]
$$

describes a bath of harmonic oscillators with mass $m_{\xi}$, frequency $\omega_{\xi}$, and momentum $p_{\xi}$, shifted due to the coupling with the exciton state $|\mu\rangle$.

In addition to the redefinition of the system and the bath Hamiltonian, one has to define a different type of projection operator, which only projects on the diagonal part of the system density matrix in the eigenstate basis. This is achieved by

$$
\widetilde{P}=\sum_{\mu=0}^{N} P_{\mu} \quad \text { with } \quad P_{\mu} \cdot=R_{e q}^{\mu} \operatorname{tr}\{|\mu\rangle\langle\mu| \cdot\},
$$

where $P_{\mu}$ is the projector onto the $\mu$ th excitonic state and $R_{e q}^{\mu}=\exp \left[-\beta H_{B}(\mu)\right] / Z_{e q}^{\mu}$ is the equilibrium density matrix of the bath when the system is in the excitonic state $|\mu\rangle$. Here, $Z_{e q}^{\mu}=\operatorname{tr} \exp \left[-\beta \mathrm{H}_{\mathrm{B}}(\mu)\right]$ with $\beta=1 /\left(k_{B} T\right)$ and $T$ being the temperature.

Inserting these definitions into the Nakajima-Zwanzig equation, determining $H^{\prime}$ up to second order and invoking the time-dependent population transfer rate, one obtains an equation of motion for the population transfer terms in the form

$$
\frac{\partial}{\partial t} \rho_{\mu \mu}(t)=\sum_{\nu \neq \mu}\left[R_{\mu \mu \nu v}(t) \rho_{\nu v}(t)-R_{\nu \nu \mu \mu}(t) \rho_{\mu \mu}(t)\right],
$$


with the population transfer rates [20]

$$
\begin{aligned}
& R_{\mu \mu \nu \nu}(t)=2 \operatorname{Re} \int_{0}^{t} d \tau \operatorname{tr}\left\{|\nu\rangle\left\langle\nu\left|\exp \left(-i H_{0} \tau\right) H^{\prime}\right| \mu\right\rangle\langle\mu|\right. \\
& \left.\times R_{e q}^{\mu} \exp \left(i H_{0} \tau\right) H^{\prime}\right\} \\
& =2 \operatorname{Re} \int_{0}^{t} d \tau \exp \left[-i \omega_{\mu \nu} \tau-g_{\mu \mu \mu \mu}(\tau)-g_{\nu \nu \nu v}(\tau)\right. \\
& \left.+g_{\nu \nu \mu \mu}(\tau)+g_{\mu \mu \nu v}(\tau)-2 i\left(\lambda_{\nu \nu \nu v}-\lambda_{\mu \mu \nu v}\right) \tau\right] \\
& \times\left\{\ddot{g}_{\mu \nu \nu \mu}(\tau)-\left[\dot{g}_{\nu \mu \nu v}(\tau)-\dot{g}_{\nu \mu \mu \mu}(\tau)+2 i \lambda_{\nu \mu \nu \nu}\right]\right. \\
& \left.\times\left[\dot{g}_{\nu \nu \mu \nu}(\tau)-\dot{g}_{\mu \mu \mu \nu}(\tau)+2 i \lambda_{\mu \nu v v}\right]\right\} .
\end{aligned}
$$

Here, $\omega_{\mu \nu}=\epsilon_{\mu}-\epsilon_{\nu}$. The line-shape function $g_{\mu \nu \mu^{\prime} \nu^{\prime}}(t)$ can be written as the two-time integral of the bath correlation function according to

$$
\begin{aligned}
g_{\mu \nu \mu^{\prime} \nu^{\prime}}(t) & =\sum_{k}\left\langle\mu\left|K_{k}\right| \nu\right\rangle\left\langle\mu^{\prime}\left|K_{k}\right| v^{\prime}\right\rangle \int_{0}^{t} d \tau \int_{0}^{\tau} d \tau^{\prime} C\left(\tau^{\prime}\right), \\
\text { with } C(t) & =\int_{-\infty}^{\infty} \frac{d \omega}{\pi} J(\omega) \frac{e^{i \omega t}}{e^{\beta \omega}-1} .
\end{aligned}
$$

To obtain Eq. (14), we have used the cumulant expansion up to second order in the system-bath coupling and have taken the independent bath model into account. The absorption line shape within the CMRT is given by

$$
\begin{aligned}
I(\omega)= & \operatorname{Re} \sum_{\mu} d_{\mu} \int_{0}^{\infty} d t \\
& \times \exp \left[i\left(\omega-\omega_{\mu 0}\right) t-g_{\mu \mu \mu \mu}(t)-\frac{1}{2} \sum_{\nu \neq \mu} \int_{0}^{t} R_{\mu \mu \nu \nu}(\tau)\right] .
\end{aligned}
$$

as detailed in Ref. [28].

Up to this point, Eq. (13) constitutes the modified Redfield theory, as developed and applied in Refs. [20-26]. Based on the population transfer term in Eq. (13), we extend the quantum master equation by including also the coherence (or, off-diagonal) terms of the reduced density matrix. The resulting coherent modified Redfield quantum master equation now reads [40]

$$
\frac{\partial}{\partial t} \rho(t)=-i[H+F(t), \rho(t)]-\Re\{\rho(t)\},
$$

where $F(t)$ is the time-dependent system-field interaction term.

The relaxation and dephasing operator $\mathfrak{R}\{\rho(t)\}$ now also includes diagonal and off-diagonal terms. The diagonal part of the relaxation operator, which was described in Ref. [41], reads

$$
\Re\{\rho(t)\}_{\mu \mu}=\sum_{\nu \neq \mu}\left[R_{\mu \mu \nu v}(t) \rho_{\nu v}-R_{\nu v \mu \mu}(t) \rho_{\mu \mu}\right] .
$$

The off-diagonal terms $\Re\{\rho(t)\}_{\mu \nu}$ are now included in order to describe decoherence of excited states and electronic dephasing between the ground and excited states. Here, we use an efficient way to obtain the associated rates by exploiting the relation $1 / T_{2}=1 / 2 T_{1}+1 / T_{2}^{*}$ to estimate the different contributions to the dephasing rate. $T_{2}$ is the transverse relaxation time, $T_{1}, T_{2}^{*}$ are the longitudinal relaxation time and pure dephasing time, respectively [42]. In detail, $1 / T_{1}=$ $\sum_{e \neq \mu} R_{\mu \mu e e}+\sum_{e \neq v} R_{v v e e}$ and $1 / T_{2}^{*}$ is given by the first derivative of line-shape function $g_{\mu \mu \nu v}(t)$. Therefore, the off-diagonal terms of the excited states and between the ground and excited states can be written as

$$
\begin{aligned}
\Re\{\rho(t)\}_{\mu \nu}= & \left\{\frac{1}{2}\left[\sum_{e \neq \mu} R_{\mu \mu e e}(t)+\sum_{e \neq \nu} R_{v v e e}(t)\right]\right. \\
& \left.+\dot{g}_{\mu \mu \nu v}(t)\right\} \rho_{\mu \nu}(t), \\
\Re\{\rho(t)\}_{\mu 0}= & \left\{\frac{1}{2}\left[\sum_{m \neq n} R_{m m n n}(t)+\sum_{n \neq m} R_{n n m m}(t)\right]\right. \\
& \left.+\dot{g}_{\mu \mu \mu \mu}(t)\right\} \rho_{\mu 0}(t) .
\end{aligned}
$$

This extended quantum master equation has also been independently put forward very recently in Ref. [30] and has been named the coherent modified Redfield theory (CMRT). It is an efficient, but approximate way to take into account population transfer and dephasing on the same footing.

\section{B. QUAPI}

The quasiadiabatic propagator path integral $[43,44]$ is a numerically exact approach to determine the influence of environmental fluctuations on the system dynamics within an open quantum systems approach. Specifically, QUAPI determines the time-dependent reduced statistical operator $\rho(t)$ of the system. It is well established in the literature and we only briefly summarize the central features in the following. The algorithm is based on a symmetric Trotter splitting of the short-time propagator $\mathcal{K}\left(t_{k+1}, t_{k}\right)$ for the full Hamiltonian into two parts, one depending on the system Hamiltonian, and one involving the bath and the coupling term. The short-time propagator determines the time evolution over a Trotter time slice $\delta t$. The discrete time evolution becomes exact in the limit $\delta t \rightarrow 0$. For any finite $\delta t$, a finite Trotter error occurs, which has to be eliminated by choosing $\delta t$ small enough to achieve convergence. On the other side, the environmental degrees of freedom generate correlations, which are nonlocal in time. For any finite temperature, these correlations decay on a time scale denoted as the memory time scale. The QUAPI scheme defines an augmented reduced density tensor, which lives on this full memory time window. Then, an iteration scheme is established in order to extract the time evolution of this object. All correlations are completely included over the finite memory time $\tau_{\text {mem }}=K \delta t$ but are neglected for times beyond $\tau_{\text {mem }}$. One increases the memory parameter $K$ until convergence is found. The two requirements to achieve convergence, i.e., minimize $\delta t$ but maximize $\tau_{\text {mem }}=K \delta t$, are naturally opposed to each other, but nevertheless convergent results can be obtained in a wide range of parameters, including the cases presented in this work. 


\section{Two-dimensional electronic spectroscopy}

In the equation of motion phasing matching approach (PMA), the polarization in the photon-echo direction is calculated by simultaneously propagating three auxiliary density matrices $\rho_{1}, \rho_{2}$ and $\rho_{3}$, thereby employing also the rotating wave approximation [36]. The time evolution equations are given by

$$
\begin{aligned}
\frac{\partial}{\partial t} \rho_{1}(t)= & -i\left[H-V_{1}\left(t, t_{1}\right)-V_{2}^{\dagger}\left(t, t_{2}\right)-V_{3}^{\dagger}\left(t, t_{3}\right), \rho_{1}(t)\right] \\
& -\Re\left\{\rho_{1}(t)\right\}, \\
\frac{\partial}{\partial t} \rho_{2}(t)= & -i\left[H-V_{1}\left(t, t_{1}\right)-V_{2}^{\dagger}\left(t, t_{2}\right), \rho_{2}(t)\right]-\Re\left\{\rho_{2}(t)\right\}, \\
\frac{\partial}{\partial t} \rho_{3}(t)= & -i\left[H-V_{1}\left(t, t_{1}\right)-V_{3}^{\dagger}\left(t, t_{3}\right), \rho_{3}(t)\right]-\Re\left\{\rho_{3}(t)\right\} .
\end{aligned}
$$

where $\quad V_{\alpha}=X E_{\alpha}\left(t-t_{\alpha}\right) e^{i \omega t} \quad$ and $\quad E_{\alpha}\left(t-t_{\alpha}\right)=$ $\exp \left[-4 \ln 2\left(t-t_{\alpha}\right)^{2} / \tau_{p}^{2}\right], \quad \tau_{p}$ is the pulse duration. To obtain the third-order $2 \mathrm{D}$ signal, the polarization in the phase matching direction is evaluated as

$$
P_{P E}\left(t_{1}, t_{2}, t_{3}\right)=e^{i \mathbf{k}_{s} \cdot \mathbf{r}}\left\langle X\left[\rho_{1}(t)-\rho_{2}(t)-\rho_{3}(t)\right]\right\rangle+\text { c.c. },
$$

where the bracket $\langle\ldots\rangle$ denotes the trace. Experimentally, in the limit of ideal detection, the heterodyne photon-echo signal is proportional to the polarization $P_{P E}\left(t_{1}, t_{2}, t_{3}, t\right)$, where $t$ is the detection time. Therefore, the ideal total $2 \mathrm{D}$ signal can be expressed as

$$
S_{T}\left(\omega_{\tau}, T, \omega_{t}\right) \propto \int_{-\infty}^{\infty} d \tau \int_{-\infty}^{\infty} d t e^{-i \omega_{\tau} \tau} e^{i \omega_{t} t} i P_{P E}(\tau, T, t),
$$

where $\tau, T$, and $t$ denote coherence time, population (waiting) time and detection time, respectively, $\tau=t_{2}-t_{1}, T=t_{3}-$ $t_{2}$. The coherence time corresponds to a period in which the system is coherently evolving after the first interaction with the optical field. The second interaction with the field creates population states and the third interaction recovers the coherence again. The Fourier transform in Eq. (22) is always performed over the coherence time $\tau$ and the detection time $t$. The corresponding frequencies $\omega_{\tau}, \omega_{t}$ are often referred to as absorption and emission frequencies, respectively. In addition, Gaussian laser pulses have been assumed for a realistic detection scheme, which have the form

$$
\mathbf{E}(t)=\sum_{\alpha=1}^{3} A e^{-4 \ln 2\left(t-t_{\alpha}\right)^{2} / \tau_{p}^{2}} e^{i \omega t} e^{-i \mathbf{k}_{\alpha} \mathbf{r}}+\text { c.c. }
$$

where $A, t_{\alpha}, \mathbf{k}_{\alpha}$, and $\omega$ are the amplitude, envelop central time, wave vector, and frequency of the pulses and $\tau_{p}$ characterizes the pulse duration. In order to obtain the undisturbed photonecho signal, a relatively small laser pulse strength $A$, compared to the parameters in the molecular Hamiltonian $H_{S}$, has to be used. In addition, we would like to point out that another useful method to compute nonlinear spectra exists, which does not suffer from this problem [45]. Note that all the pulses are assumed to have the same line shape, carrier frequencies, and durations in this paper.

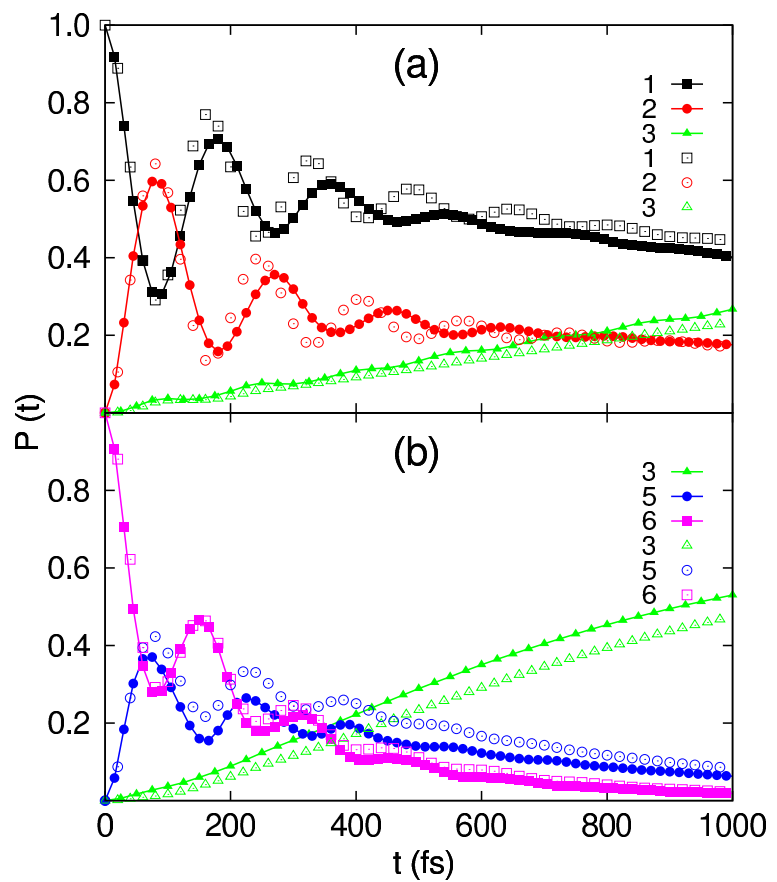

FIG. 1. (Color online) Population dynamics of selected FMO sites. In (a), the population of sites 1 (square), 2 (circle), and 3 (triangle) with the initial condition $\rho(1,1)=1$ is shown, while in (b) the populations of sites 3 (triangle), 5 (circle), and 6 (square) with the initial condition $\rho(6,6)=1$ is depicted (symbols: QUAPI, full lines with points: CMRT) for the parameters as given in the text.

\section{RESULTS}

\section{A. Population dynamics of the FMO complex}

In order to verify the reliability of the CMRT, we present the population dynamics of the FMO complex calculated by CMRT and compare the results to those obtained by the numerically exact QUAPI method. In Fig. 1, the population dynamics of selected FMO sites is shown for $T=77 \mathrm{~K}$ for two different initial conditions. In Fig. 1(a), we assume the energy transfer to start from site 1 . We monitor then the full transfer, which involves all seven FMO sites. For simplicity, we only show the population dynamics of the sites 1,2 , and 3. Alternatively, the energy transfer may be assumed to start from site 6, see Fig. 1(b). There, we depict the population dynamics of the relevant sites 3,5 , and 6 . We observe that the oscillatory behavior of the populations is captured by both approaches. Both also yield the same decay rates and periods of oscillations. However, a phase shift of the oscillations occurs between the CMRT and QUAPI results. Energy transfer is believed to be related to the population of the FMO site 3 (green symbols and lines), which has the lowest energy in the FMO monomer. In our comparison, CMRT slightly overestimates the population transfer efficiency towards site 3 . All in all, the CMRT results for the FMO exciton population dynamics are in good agreement to numerically exact QUAPI results. Since the system-bath coupling parameters of the FMO complex are typical for natural photosynthetic units, we conclude that CMRT is a useful tool to study their exciton dynamics. 


\section{B. Two-dimensional spectra of a dimer system}

To obtain 2D spectra, we combine the CMRT next with the PMA. This constitutes a very efficient approximate numerical tool whose reliability is assessed by a comparison with $2 \mathrm{D}$ spectra obtained by QUAPI. Since 2D spectra involve extended numerical calculations, QUAPI results are available only for small model systems with present day hardware technology. For such a comparison, we present the calculated results for the dimer model. It allows us to study energy transfer and dephasing (homogeneous broadening) as building blocks of the exciton dynamics in larger molecular compounds. It can still be treated by QUAPI with reasonable numerical effort.

Figure 2 (left) shows 2D spectra of the dimer calculated by CMRT+PMA for $\lambda=50 \mathrm{~cm}^{-1}$ and the other parameters as indicated above. They are compared to QUAPI results (right column in Fig. 2) for waiting times $T=0 \mathrm{fs}$, 50fs, 100fs, and 500fs. These 2D spectra show two diagonal peaks (labeled A, B), which correspond to the two exciton states. Moreover, two cross peaks (labeled C and D) arise due to the excitonic coupling between them. For the sake of simplicity and clarity of the comparison, inhomogeneous broadening and the rotational averaging for different laser polarizations and molecular orientations is not performed here. Although this would be important to describe realistic experimental situations, the averaged results generally show smaller discrepancies (not shown).

At $T=0$ fs, the two results show the same profile for diagonal and cross peaks and, indeed, the agreement is excellent. This shows that the CMRT correctly models the coherence times and the system-bath correlations created during the simulation. With increasing waiting time, the same coherent dynamics is found for both the diagonal and the cross peaks and even can be inspected by eye. However, some disagreement is observed at long waiting time $T=500 \mathrm{fs}$. The diagonal peak $\mathrm{B}$ in left figure (CMRT+PMA) shows a somewhat reduced amplitude as compared to the right figure (QUAPI).

For a more refined comparison, the amplitudes of the diagonal and cross peaks (A, B, C, and D) are plotted against the waiting time in Figs. 3 and 4 . In Fig. 3, the population dynamics of the diagonal peaks A (top) and B (bottom) calculated by CMRT+PMA from 0-1000fs is shown and compared to the QUAPI result. We find that the CMRT+PMA provides reasonably accurate results for the population transfer and the oscillation period. However, the amplitude of peak B decays slightly faster in the approximate results as compared to the QUAPI data. Moreover, both yield different stationary states. In addition, the phase of the oscillations is slightly shifted. For the comparison of the cross peaks, the oscillatory behavior of peaks $\mathrm{C}$ and $\mathrm{D}$ is plotted in Fig. 4. Cross peak $\mathrm{C}$ shows a similar oscillatory behavior but the two approaches yield different stationary states. Peak D shows a only slightly shifted phase of the oscillatory behavior. Such a phase shift was also observed in the population dynamics of the FMO complex shown above. The phase shift might be due to the neglect of imaginary parts in the Redfield relaxation tensor.

In order to further assess the reliability of the CMRT+PMA, we have repeated the calculations for a larger reorganization energy, i.e., for $\lambda=100 \mathrm{~cm}^{-1}$ (with $\gamma=100 \mathrm{~cm}^{-1}$

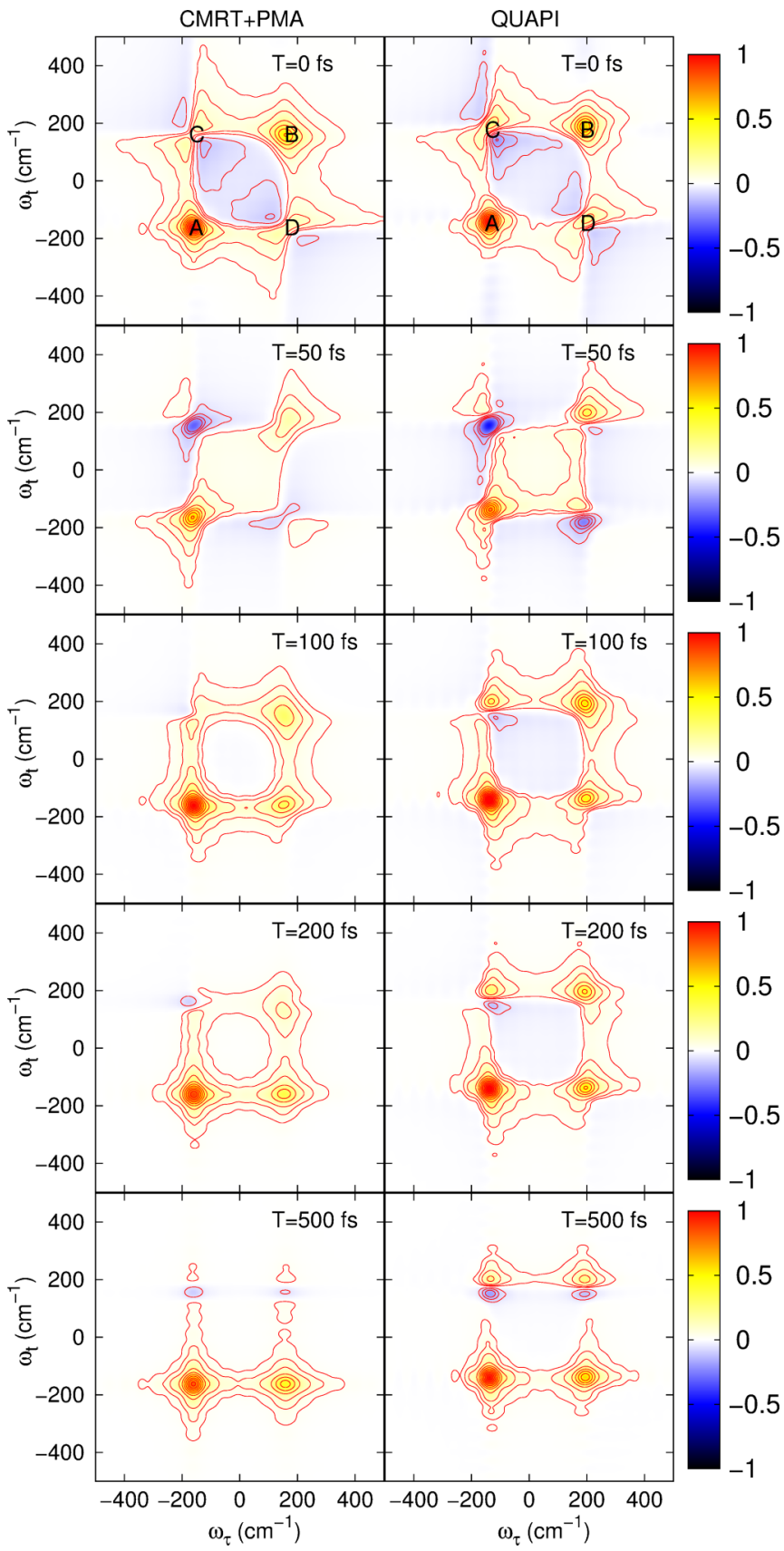

FIG. 2. (Color online) Two-dimensional photon-echo spectra of a dimer model calculated by CMRT+PMA (left) and QUAPI (right) for different waiting times as indicated. The Debye spectral density was used for the calculation with the parameters $\lambda=50 \mathrm{~cm}^{-1}, \gamma=$ $100 \mathrm{~cm}^{-1}$ and the temperature was set to $T=77 \mathrm{~K}$.

kept unchanged). 2D spectra were again calculated by both approaches and the amplitude of the labeled peaks were extracted. Their time dependence is plotted in Figs. 5 and 6. CMRT+PMA still yields quantitative agreement with the QUAPI result except for the behavior of the damping. The stronger system-bath coupling results in faster damping (diagonal peak A) and also in an increased difference between QUAPI and CMRT+PMA as compared to the weaker coupling with $\lambda=50 \mathrm{~cm}^{-1}$. 


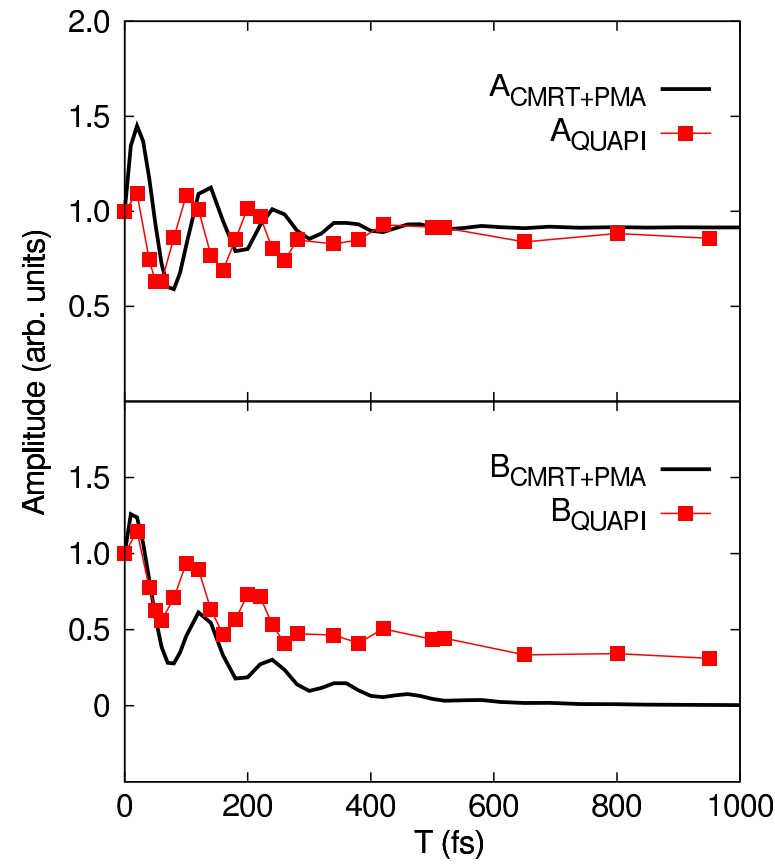

FIG. 3. (Color online) Population dynamics of the labeled diagonal peaks (A, B) extracted from the underlying sequence of 2D maps. The two approaches yield the same oscillation period. The diagonal peak B obtained from CMRT+PMA decays faster as compared to the QUAPI result. The oscillation periods can be extracted by data fitting and are: CMRT+PMA: 110fs, QUAPI: 99fs.

\section{DISCUSSION: CMRT+PMA VERSUS QUAPI}

From the above comparison of the results obtained by both approaches, we observe that the discrepancies found in the $2 \mathrm{D}$ calculations are more pronounced than in the dynamics of the populations. Put differently, nonlinear 2D spectra are more sensitive to assess the performance and reliability of approximate theoretical approaches. In order to understand this, we point out two fundamental differences between 2D spectra and the population dynamics. First, entanglement between the system and the bath leads to initial correlations at the beginning of the waiting time window, which are absent in the calculation of the population dynamics. Second, two-exciton states contribute to the 2D spectra during the detection time, and interference between positive and negative peaks changes the observed amplitudes. This shows that one cannot understand the reliability of a method to simulate correct 2D spectra by calculating population dynamics alone. Our current framework, in which we use the combined CMRT+PMA and compare the results with QUAPI, is well suited to show the performance of these methods in understanding 2D spectra directly.

In more detail, we have observed three noticeable discrepancies of the CMRT+PMA as compared to QUAPI: (i) shifted oscillation phase of peak intensities, (ii) a slightly faster decay, and, (iii) a different amplitude of peaks B and C for long waiting times.

For the explanation of the shifted oscillation observed in 2D simulations of the CMRT+PMA, we need to notice that Eqs. (18), (19) provide the analytic result for a monomer (two-level system), and that this has been proven by comparing

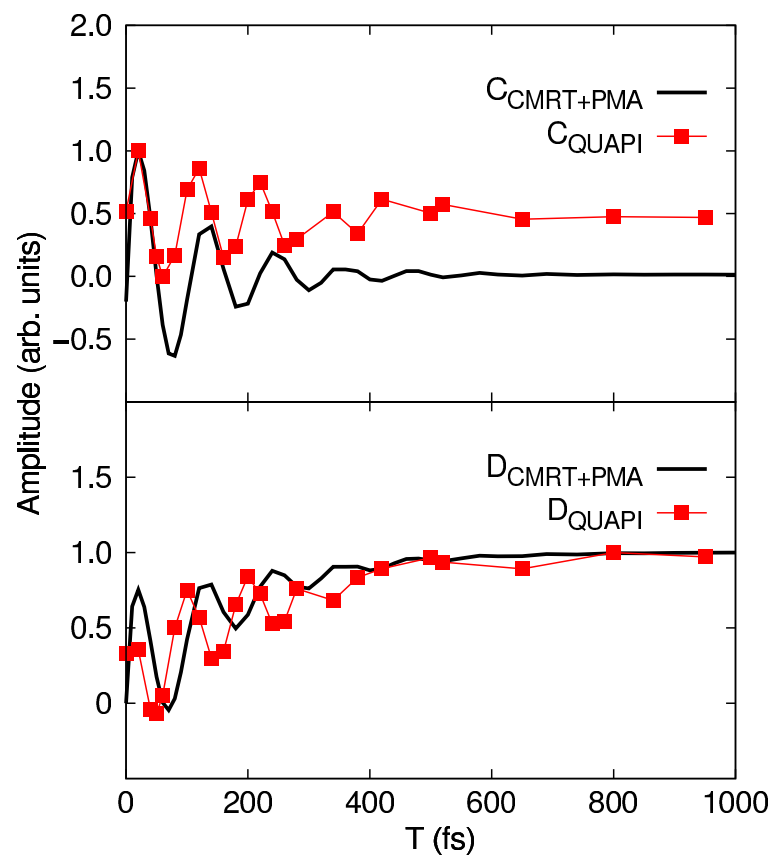

FIG. 4. (Color online) Coherent oscillations of labeled cross peaks (C, D) extracted from 2D maps. Cross peak $\mathrm{C}$ obtained by CMRT+PMA shows the same oscillatory behavior, but with a somewhat smaller amplitude.

to QUAPI [46]. However, CMRT yields a shifted period for the dimer model. The mismatch is mainly caused by the population transfer term $R(t)$ since there is no population transfer term in the monomer model. In this paper, the population

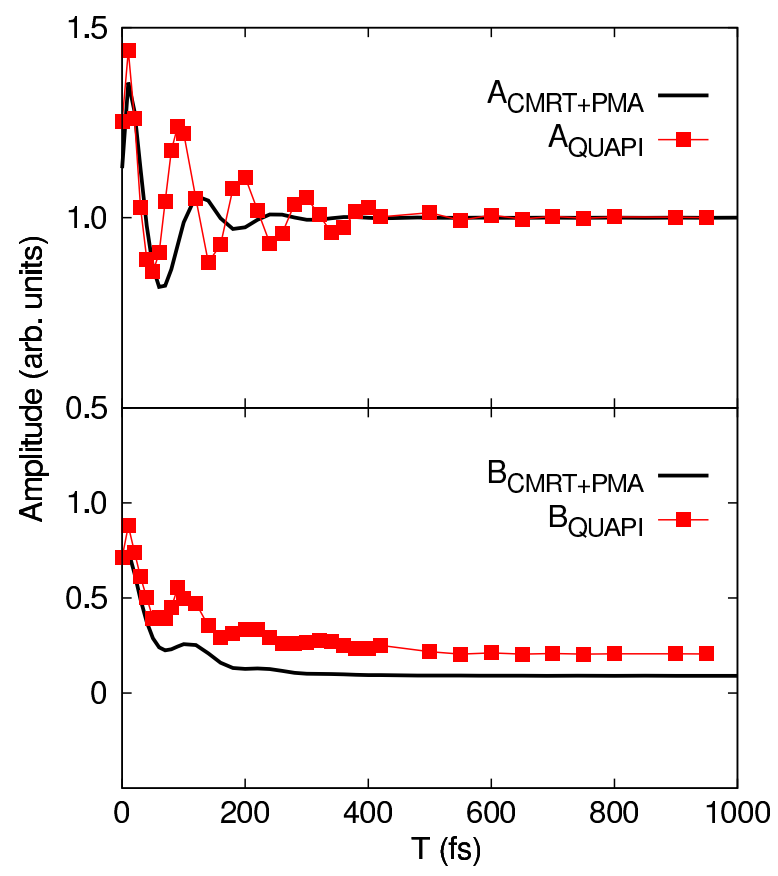

FIG. 5. (Color online) Amplitude of the diagonal peaks A and B for a stronger system-bath coupling $\lambda=100 \mathrm{~cm}^{-1}$ (with $\gamma=$ $100 \mathrm{~cm}^{-1}$ unchanged). CMRT+PMA calculations yield a faster decay (A) as compared to the QUAPI result (decay rate extracted from a fit: CMRT+PMA: 81fs, QUAPI: 146fs). 


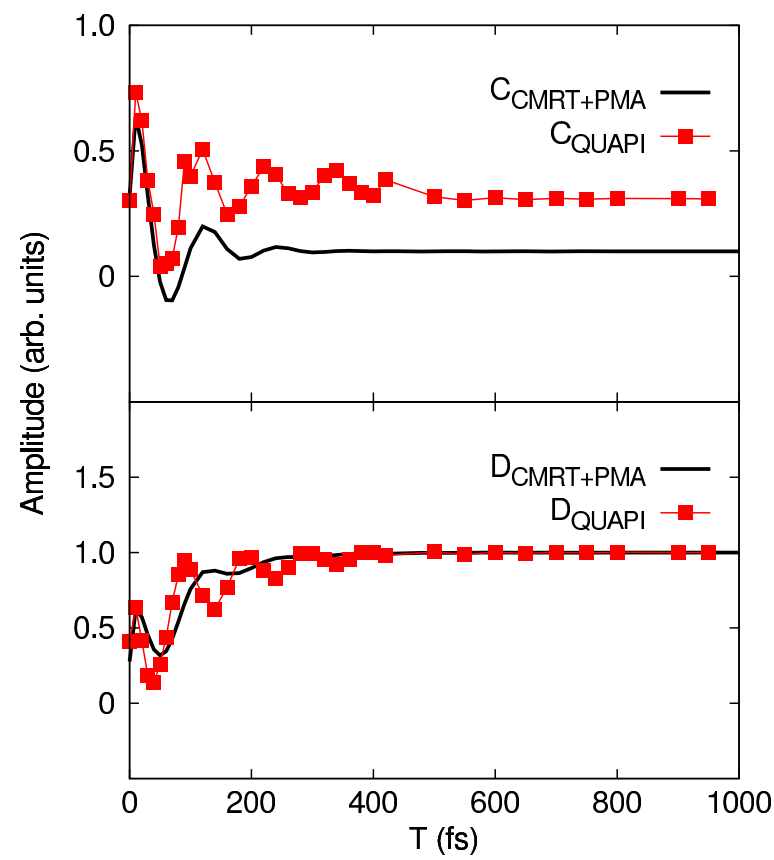

FIG. 6. (Color online) Oscillations of the cross peaks (C, D) for a stronger system-bath interaction (same parameters as in Fig. 5). The cross peak $\mathrm{C}$ calculated by CMRT + PMA yields a faster decay and a smaller amplitude (decay rates as obtained from a fit: CMRT+PMA: 62fs, QUAPI: 177fs).

transfer rate was calculated by the cumulant expansion in Eq. (14) [20], which is derived based on the second-order perturbation approximation. It is one of the reasons for the explanation of the oscillatory shift and slightly fast decay of the oscillations found in CMRT calculations. Furthermore, the secular approximation was used to separate the population dynamics and the dephasing process in Eqs. (18), (19). This also contributes to the discrepancy in the decay rate, since it neglects the interference between population transfer and coherence dephasing. Besides, the imaginary part of $\dot{g}(t)$ is important for the spectral calculation, since it contains the information of the reorganization energy [47].

A relatively small amplitude of peak B and C was found in Fig. 3 and Fig. 4 and it also can be observed by eye in the 2D map for the long waiting time $T=500 \mathrm{fs}$. We observe that peaks $\mathrm{B}$ and $\mathrm{C}$ are mainly formed by one positive (red) peak and overlap with a negative (blue) peak in the 2D spectrum ( $T=500 \mathrm{fs}$ ). Therefore, the amplitude of those peaks mainly depends on the overlap of two peaks. In the QUAPI result, the two peaks are clearly separated with a larger spectral distance than in the CMRT result and this leads to the larger amplitude of peaks B and C in the 2D spectrum calculated with QUAPI. It indicates that, besides the shifted oscillation and the faster decay of the oscillation, CMRT does not properly account for the reorganization energy by the heat bath (diagonal peaks show slightly different positions in the 2D map: $-190 \mathrm{~cm}^{-1}$ and $190 \mathrm{~cm}^{-1}$ for CMRT and $-180 \mathrm{~cm}^{-1}$ and $200 \mathrm{~cm}^{-1}$ for QUAPI). In the CMRT, the reorganization energy is included in the diagonal part of the Hamiltonian by Eq. (3), where it just brings in a shift of the excitonic transition frequency $E_{\mu}$ by the renormalization term Eq. (3) and does not affect the dynamics of the off-diagonal terms in the Hamiltonian in Eq. (8).
On the basis of a clear physical meaning (population transfer and dephasing terms) and for the purpose of an efficient and fast calculation, the secular approximation and the second-order perturbation theory were applied to construct the CMRT. On the one hand, the secular approximation leads to a separation of the population dynamics and dephasing process and avoids any complicated interaction terms between diagonal and off-diagonal parts in the equation of motion. On the other hand, the second-order perturbation theory simplifies the population transfers. It is possible to improve the equation by including higher orders. However, this renders the equation considerably more complicated and requires more computational resources for the simulation and it is a priori unclear how much this improves the accuracy.

\section{CONCLUSIONS}

In this paper we present the CMRT and compare it in more detail to the QUAPI method by calculating the population dynamics of selected FMO exciton sites and the 2D spectrum of a model dimer. We found that CMRT provides numerically reliable results as compared to numerically exact QUAPI calculations for both the population dynamics and 2D spectra, as long as the reorganization energy is not too large compared to the typical energy gap of the system. Most importantly, it requires smaller computational efforts and orders of magnitude shorter calculation times. It provides us with an efficient approach to study the energy transfer in superlarge molecular complexes and to perform complicated 2D simulations.

We found that the 2D profile calculated from CMRT+PMA agrees well with the corresponding QUAPI results. For a quantitative comparison, the amplitudes of diagonal and cross peaks were extracted from 2D maps and compared to those calculated by QUAPI. Quantitative agreement was found. We observe some discrepancies. In particular, oscillations are shifted, they decay slightly faster, and positions of peaks are slightly shifted. This becomes more serious if the reorganization energy is increased, which is also the case in Refs. [48,49].

The simulation protocol developed here can be used for arbitrary forms of the spectral density. For instance, the spectral density obtained from quantum mechanics and molecular mechanics [50,51] is rather involved and contains a rich structure due to vibrational contributions. One can envision an approach where the CMRT method is used to simulate superlarge complexes, while numerically exact methods such as QUAPI play a role in benchmarking the accuracy of the simulations of smaller systems.

\section{ACKNOWLEDGMENTS}

We acknowledge financial support by the Joachim-HerzStiftung, Hamburg within the PIER Fellowship program (HGD) and by the excellence cluster "The Hamburg Center for Ultrafast Imaging - Structure, Dynamics and Control of Matter at the Atomic Scale" of the Deutsche Forschungsgemeinschaft. A.G.D. was supported by a Marie Curie International Incoming Fellowship within the 7th European Community Framework Programme. P.N. acknowledges financial support by the DFG project NA394/2-1. 
[1] R. E. Blankenship, Molecular Mechanisms of Photosynthesis (Blackwell Science, Malden, Massachusetts, 2002).

[2] V. I. Prokhorenko, A. Halpin, and R. J. D. Miller, Opt. Express 17, 9764 (2009).

[3] G. S. Engel, T. R. Calhoun, E. L. Read, T.-K. Ahn, T. Mančal, Y.-C. Cheng, R. E. Blankenship, and G. R. Fleming, Nature (London) 446, 782 (2007).

[4] E. Collini, C. Y. Wong, K. E. Wilk, P. M. G. Curmi, P. Brumer, and G. D. Scholes, Nature (London) 463, 644 (2010).

[5] D. Zigmantas, E. L. Read, T. Mančal, T. Brixner, A. T. Gardiner, R. J. Cogdell, and G. R. Fleming, Proc. PNAS 103, 12672 (2006).

[6] E. Romero, R. Augulis, V. I. Novoderezhkin, M. Ferretti, J. Thieme, D. Zigmantas, and R. van Grondelle, Nature Phys. 10, 676 (2014).

[7] F. D. Fuller, J. Pan, A. Gelzinis, V. Butkus, S. S. Senlik, D. E. Wilcox, C. F. Yocum, L. Valkunas, D. Abramavicius, and J. P. Ogilvie, Nature Chem. 6, 706 (2014).

[8] A. Ishizaki and G. R. Fleming, Proc. Natl. Acad. Sci. USA 106, 17255 (2009).

[9] M. B. Plenio, J. Almeida, and S. F. Huelga, J. Chem. Phys. 139, 235102 (2013).

[10] P. Nalbach, D. Braun, and M. Thorwart, Phys. Rev. E 84, 041926 (2011).

[11] M. Thorwart, J. Eckel, J. H. Rina, P. Nalbach, and S. Weiss, Chem. Phys. Lett. 478, 234 (2009).

[12] C. Kreisbeck and T. Kramer, J. Phys. Chem. Lett. 3, 2828 (2012).

[13] A. G. Redfield, Adv. Magn. Reson. 1, 1 (1965).

[14] J. Adolphs and T. Renger, Biophys. J. 91, 2778 (2006).

[15] A. Ishizaki and G. R. Fleming, J. Chem. Phys. 130, 234110 (2009).

[16] P. Nalbach and M. Thorwart, J. Chem. Phys. 132, 194111 (2010).

[17] C. A. Mujica-Martinez, P. Nalbach, and M. Thorwart, Phys. Rev. E 88, 062719 (2013).

[18] P. Kjellberg, B. Brüggemann, and T. Pullerits, Phys. Rev. B 74, 024303 (2006).

[19] P. Kjellberg and T. Pullerits, J. Chem. Phys. 124, 024106 (2006).

[20] W. M. Zhang, T. Meier, V. Chernyak, and S. Mukamel, J. Chem. Phys. 108, 7763 (1998).

[21] M. Yang and G. R. Fleming, Chem. Phys. 275, 355 (2002).

[22] A. Gelzinis, L. Valkunas, F. D. Fuller, J. P. Ogilvie, S. Mukamel, and D. Abramavicius, New J. Phys. 15, 075013 (2013).

[23] V. I. Novoderezhkin, A. G. Yakovlev, R. van Grondelle, and V. A. Shuvalov, J. Phys. Chem. B 108, 7445 (2004).

[24] Q. Ai, T.-C. Yen, B.-Y. Jin, and Y.-C. Cheng, J. Phys. Chem. Lett. 4, 2577 (2013).

[25] M. Cho et al., J. Phys. Chem. B 109, 10542 (2005).

[26] K. L. M. Lewis, F. D. Fuller, J. A. Myers, C. F. Yocum, S. Mukamel, D. Abramavicius, and J. P. Ogilvie, J. Phys. Chem. A 117, 34 (2013).
[27] Y. Chang and Y.-C. Cheng, J. Chem. Phys. 142, 034109 (2015).

[28] M. Schröder, U. Kleinekathöfer, and M. Schreiber, J. Chem. Phys. 124, 084903 (2006).

[29] R. van Grondelle and V. I. Novoderezhkin, Phys. Chem. Chem. Phys. 8, 793 (2006).

[30] Y.-H. Hwang-Fu, W. Chen, and Y.-C. Cheng, Chem. Phys. 447, 46 (2015).

[31] S. Mukamel, Principles of Nonlinear Optical Spectroscopy (Oxford University Press, New York, 1995).

[32] O. Rancova and D. Abramavicius, J. Phys. Chem. B 118, 7533 (2014).

[33] G. S. Schlau-Cohen, T. R. Calhoun, N. S. Ginsberg, E. L. Read, M. Ballottari, R. Bassi, R. van Grondelle, and G. R. Fleming, J. Phys. Chem. B 113, 15352 (2009).

[34] D. Abramavicius and S. Mukamel, J. Chem. Phys. 134, 174504 (2011).

[35] L. Seidner, G. Stock, and W. Domcke, J. Chem. Phys. 103, 3998 (1995).

[36] M. F. Gelin, D. Egorova, and W. Domcke, J. Chem. Phys. 123, $164112(2005)$.

[37] L. Z. Sharp, D. Egorova, and W. Domcke, J. Chem. Phys. 132, 014501 (2010).

[38] P. Nalbach, J. Eckel, and M. Thorwart, New J. Phys. 12, 065043 (2010)

[39] C. Meier and D. J. Tannor, J. Chem. Phys. 111, 3365 (1999).

[40] J. Yuen-Zhou and A. Aspuru-Guzik, J. Chem. Phys. 134, 134505 (2011).

[41] M. Schröder, M. Schreiber, and U. Kleinekathöfer, J. Luminescence 125, 126 (2007).

[42] V. May and O. Kühn, Charge and Energy Transfer Dynamics in Molecular Systems (Wiley-VCH Press, Weinheim, 2011).

[43] N. Makri and D. E. Makarov, J. Chem. Phys. 102, 4600 (1995).

[44] N. Makri and D. E. Makarov, J. Chem. Phys. 102, 4611 (1995).

[45] J. Yuen-Zhou, J. J. Krich, I. Kassal, A. S. Johnson, and A. Aspuru-Guzik, Ultrafast Spectroscopy (IOP Publishing, Bristol, UK, 2014).

[46] We compare the absorption line shape calculated by CMRT and QUAPI. They show exactly same result.

[47] A. Ishizaki and G. R. Fleming, J. Chem. Phys. 130, 234111 (2009).

[48] J. Ma, J. M. Moix, and J. S. Cao, J. Chem. Phys. 142, 094107 (2015).

[49] J. M. Moix, J. Ma, and J. S. Cao, J. Chem. Phys. 142, 094108 (2015).

[50] C. Olbrich, J. Strümpfer, K. Schulten, and U. Kleinekathöfer, J. Phys. Chem. Lett. 2, 1771 (2011).

[51] E. Rivera, D. Montemayor, M. Masia, and D. F. Coker, J. Phys. Chem. B 117, 5510 (2013). 